\title{
Understanding the Operating Mechanism of Aqueous Pentyl Viologen/ Bromide Redox-Enhanced Electrochemical Capacitors with Ordered Mesoporous Carbon Electrodes
}

\author{
Giulio Calcagno ${ }^{1}$, Brian Evanko ${ }^{2}$, Galen D. Stucky ${ }^{2,3}$, Elisabet Ahlberg ${ }^{4}$, Seung Joon Yoo ${ }^{* 5}$, \\ and Anders E.C. Palmqvist*1
}

${ }^{I}$ Department of Chemistry and Chemical Engineering, Chalmers University of Technology, Göteborg 41296, Sweden

${ }^{2}$ Materials Department, University of California, Santa Barbara, California 93106, USA

${ }^{3}$ Department of Chemistry and Biochemistry, University of California, Santa Barbara, California 93106, USA

${ }^{4}$ Department of Chemistry and Molecular Biology, University of Gothenburg, Göteborg 41296, Sweden

${ }^{5}$ School of Materials Science and Engineering, Gwangju Institute of Science and Technology, Gwangju 61005, Republic of Korea

Corresponding Authors: sjoonyoo@gist.ac.kr, anders.palmqvist@chalmers.se
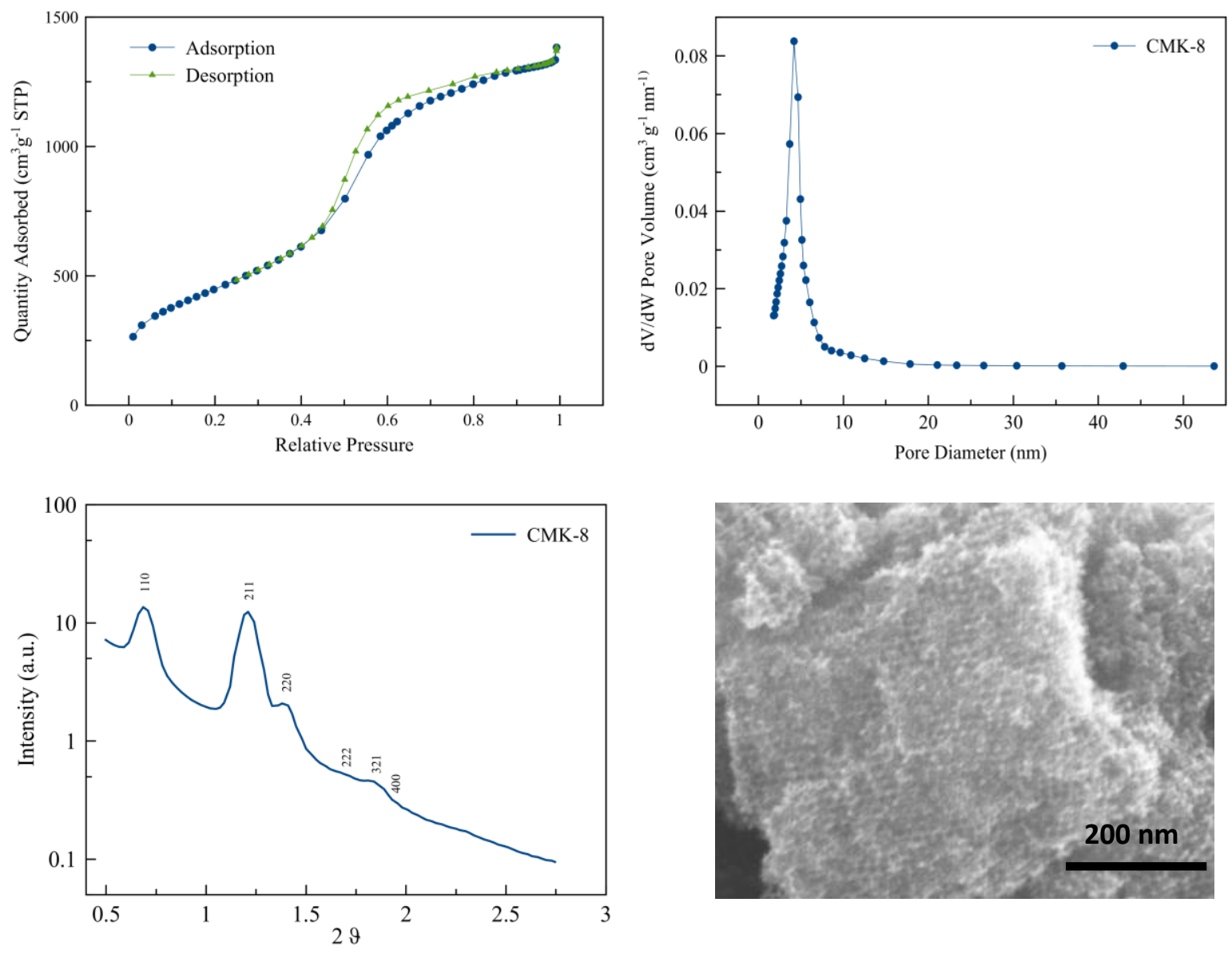
Figure S1: $N_{2}$ sorption isotherm and pore distributions (top), SAXS pattern and SEM image (bottom) for CMK-8 carbon.
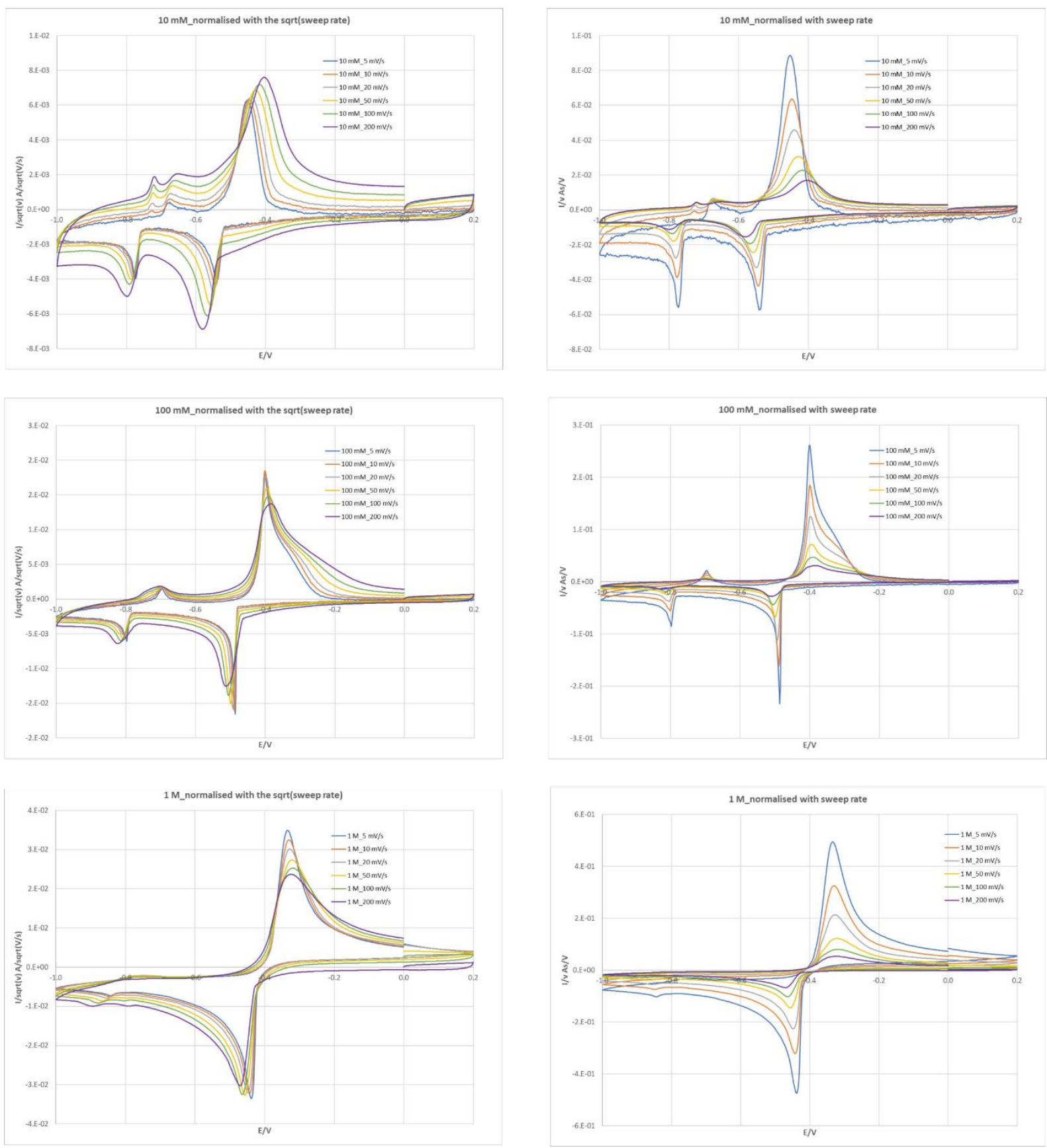

Figure S2: Normalizations of the sweep rate dependence at $10 \mathrm{mM}, 100 \mathrm{mM}$ and $1 \mathrm{M}$ pentyl viologen concentrations. The left column reports the normalization with respect to the square root of the sweep rate (diffusion-limited process). The right column reports the normalization with respect to the sweep rate (adsorption process). We can see that by increasing the concentration (from top row $(10 \mathrm{mM})$ to bottom $(1 \mathrm{M}))$ the big reduction peak at ca. $-0.45 \mathrm{~V}$ increases its adherence to a diffusion-limited process. At $1 M$ the response reflects a quasi-reversible electron transfer involving mainly solution species. 


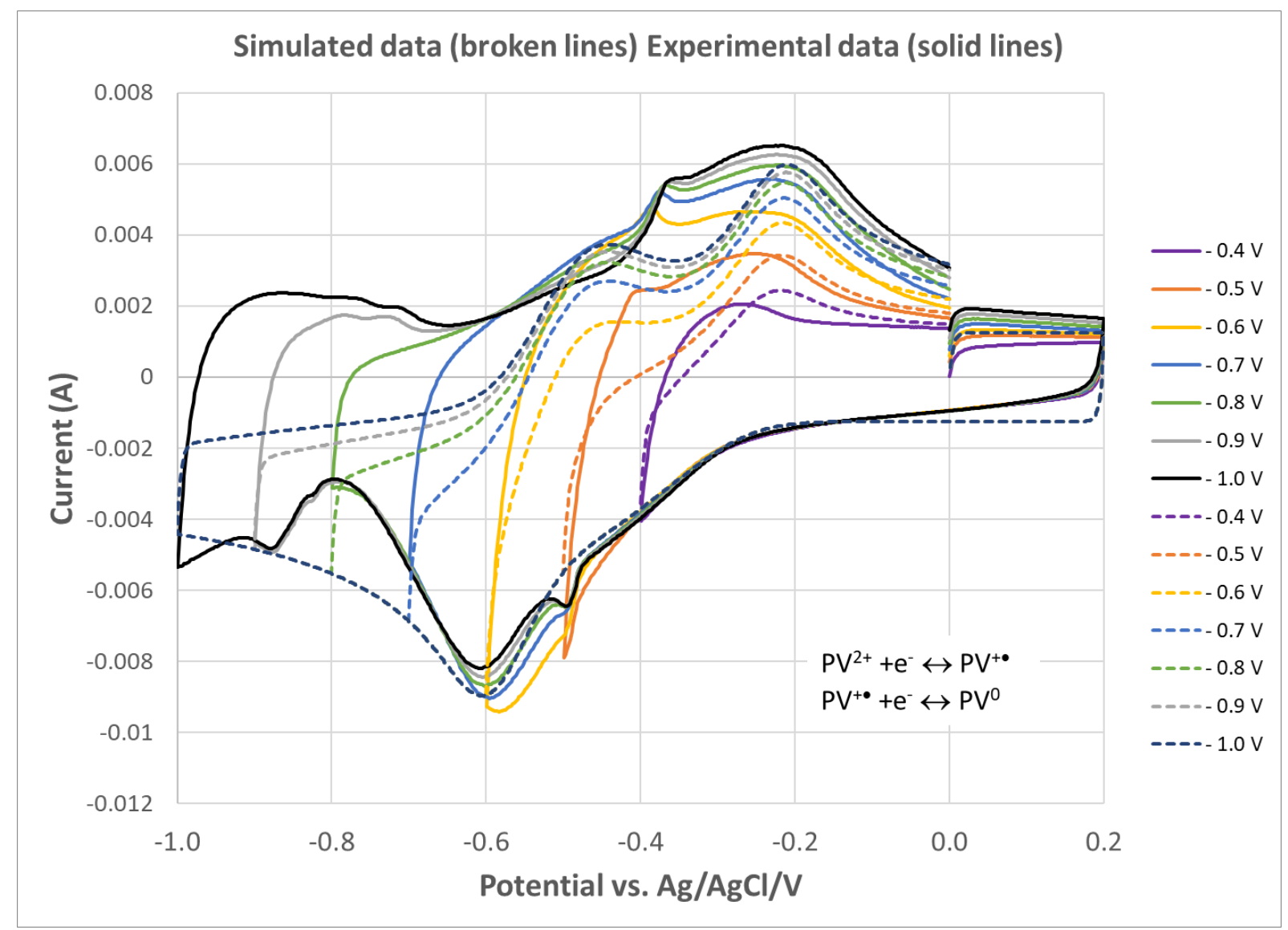

Figure S3: Simulated data (DigiElch software supplied by Gamry) for an EE redox reaction and experimental data from $C V$ testing at $5 \mathrm{mV} \mathrm{s} \mathrm{s}^{-1}$ in a three-electrode configuration with different switching potentials (-0.5 V, -0.6 V, -0.7 V, -0.8 V, -0.9 V and -1.0 V) for a CMK-8 carbon electrode. Electrolyte: $100 \mathrm{mM} \mathrm{PVBr}_{2}$ and $0.5 \mathrm{M} \mathrm{Na}_{2} \mathrm{SO}_{4}$ 


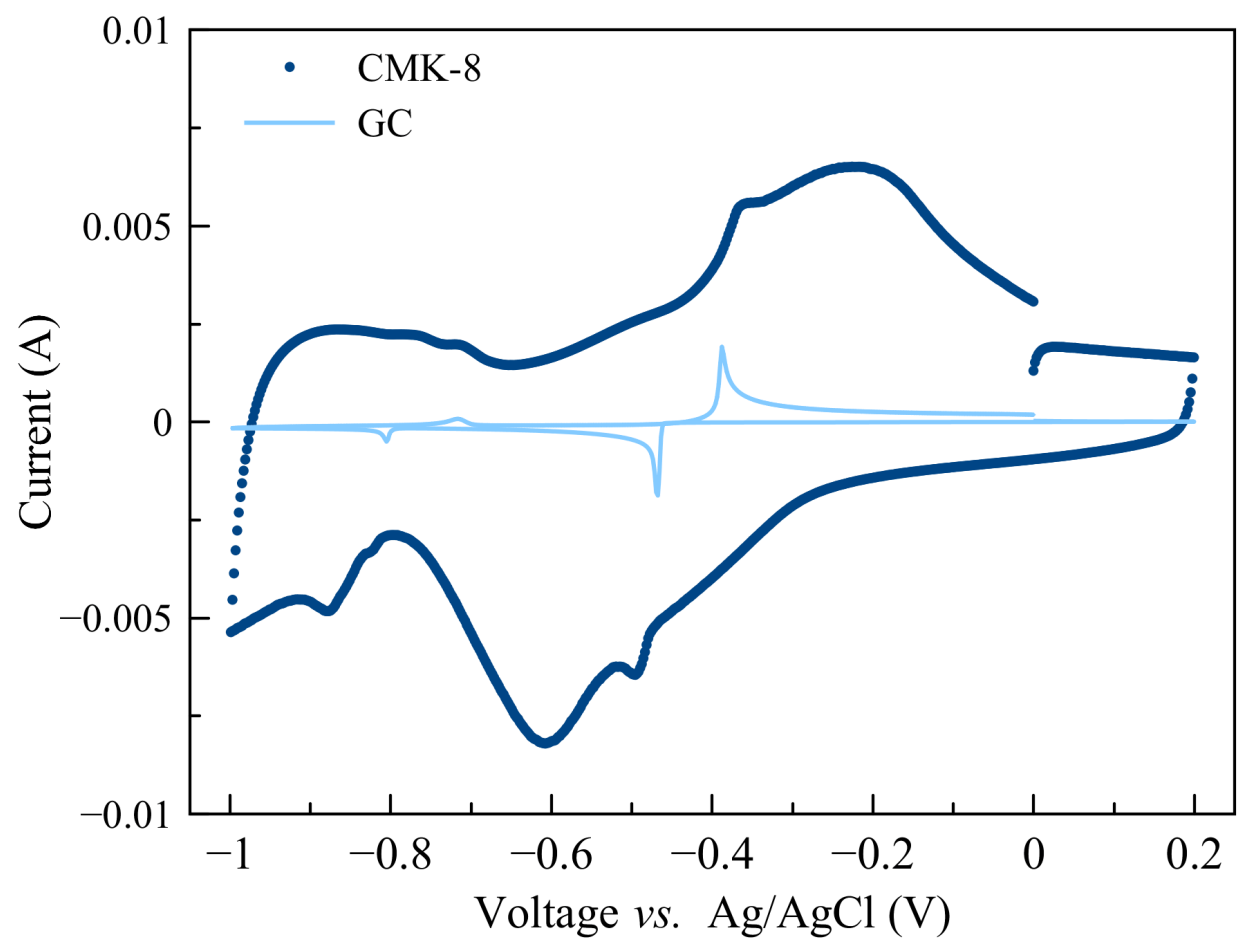

Figure S4: $C V$ testing at $5 \mathrm{mV} \mathrm{s} \mathrm{s}^{-1}$ for a CMK-8 carbon electrode (dark blue) and a glassy carbon electrode (light blue) in a three-electrode configuration. Electrolyte: $100 \mathrm{mM} \mathrm{PVBr}_{2}$ and $0.5 \mathrm{M} \mathrm{Na}_{2} \mathrm{SO}_{4}$ 